\title{
Chapter 29 \\ Starch Modification: A Model for Wheat MAS Breeding
}

\author{
Toshiki Nakamura, Patricia Vrinten, Tomoya Shimbata, and Mika Saito
}

\begin{abstract}
In the past two decades, three types of starch mutants, waxy (Wx), high amylose (HA) and sweet type wheat $(S W)$ have been developed in Japan. Each of these lines was obtained by identifying "partial" mutants with mutations in one or two homeoelogous genes derived from the A, B or D-genomes, followed by crossing to produce fully null mutants. The $W x$ line lacks the three granule-bound starch synthase I (GBSSI) proteins responsible for amylose synthesis, and HA lacks the three starch synthase IIa (SSIIa) enzymes that are involved in amylopectin synthesis. SW lacks all active GBSSI and SSIIa enzymes. "Partial" null mutants have also been used to obtain more subtle modifications in starch quality. For example, the GBSSI-B1 single null lines produce starch which is slightly reduced in amylose. This suggested that the identification of other desirable lines using MAS would allow us to further fine-tune starch characteristics. Sixty-four homozygous lines differing in GBSSI and SSIIa composition can be selected from progeny of crosses between $H A$ and Wx. Co-dominant markers for all GBSSI and SSIIa genes enabled us to select all 64 lines quickly and effectively. The modulation of starch characteristics serves as a model that demonstrates the utility of mutation identification in combination with MAS in hexaploid wheat. The availability of genome sequence information, combined with new methods of mutation detection, reduces the amount of work involved in finding single mutations in wheat, and one can easily see the potential for expanding this methodology to other traits.
\end{abstract}

\footnotetext{
T. Nakamura $(\triangle) \bullet$ M. Saito

NARO Tohoku Agricultural Research Center, Morioka 020-0198, Iwate, Japan

e-mail: tnaka@affrc.go.jp

P. Vrinten

Bioriginal Food and Science Corp, Saskatoon, SK, Canada

T. Shimbata

Central Lab. Nippon Flour Mills Co., Ltd.,

5-1-3 Midori-Gaoka, Atsugi 243-0041, Kanagawa, Japan 


\section{Introduction}

Starch is one of major components of cereal seed, and starch composition greatly influences the quality of cereal-based food products. Starch is composed of amylose, a linear chain of glucosyl units joined by $\alpha-1,4$ linkages, and amylopectin, which is a highly branched molecule with $\alpha-1,6$ linked side chains. The key enzyme in amylose synthesis is granule bound starch synthase (GBSSI, alternatively called waxy protein), and lines lacking GBSSI have amylose-free starch. In contrast, many enzymes are involved in amylopectin synthesis, including several types of starch synthases, as well as branching and debranching enzymes. Starch synthase IIa (SSIIa), which elongates the outside branches of the amylopectin molecules, appears to play a particularly important role in amylopectin synthesis.

Null mutations of the genes encoding the enzymes involved in starch synthesis can result in changes in starch structure and properties, which may affect the processing and cooking properties of starch. In diploid cereals such as maize and rice, many starch mutants have been identified and used extensively in both basic and applied research (Boyer and Hannah 2001; Vandeputte and Delcour 2004). However, although starch mutants of common wheat could potentially provide interesting new materials for the food industry, none of the typical starch mutants identified in maize and rice have been available until recently. In the past two decades, three types of starch mutants were developed in Japan; waxy $(W x, 1995)$, high amylose (HA, 1999) and sweet type wheat (SW, 2006).

\section{Waxy Wheat (GBSSI Mutant)}

The initial screening of wheat germplasm to detect genetic variation in the gene encoding GBSSI was performed using protein gel electrophoresis. When starch granule-bound proteins are run on standard SDS-PAGE gels, the waxy or GBSSI protein can be identified as a distinct band. Because wheat is a hexaploid crop, this band includes the proteins derived from the A, B and D genomes. By modifying SDS-PAGE conditions, we were able to separate the single band into three isoforms (Nakamura et al. 1993). We then used nullisomic-tetrasomic analysis to determine what genome each isoform was derived from. Germplasm screening identified several lines that were missing $W x-A 1$ and $-B 1$ proteins, and one line, Kanto107, that lacked both the $W x-A l$ and $-B l$ proteins.

These results suggested several methods for obtaining fully waxy lines. To produce tetraploid waxy wheat, Kanto 107 (K107) could be crossed with tetraploid wheat to remove the D genome. Hexaploid waxy lines could be developed by identifying a line that had a null mutation in the D gene, and combining it with K107, or alternatively mutagenic treatment of K107 could be used to inactivate the functional D gene. We were successful in selecting a fully waxy tetraploid wheat line from a 
cross between K107 and a durum wheat variety (Nakamura et al. 1995). Identification of a line with a naturally occurring mutation in the $W x-D l$ gene proved more difficult, and hundreds of lines were screened before the identification of a line (BaiHao) missing the $W x-D 1$ protein. A fully waxy hexaploid line was then selected from the progeny of a cross between Bai-Hao and K107 (Nakamura et al., 1995). Later, mutagenic treatment of K107 also resulted in the identification of a fully waxy hexaploid line (Yasui et al. 1997).

Registered varieties of waxy wheat have already been developed in several countries and many studies characterizing the chemical and rheological properties of this wheat type have been reported. Waxy wheat showed substantial differences in flour properties compared to control lines, as evidenced by rapid viscosity analyzer (RVA) and differential scanning calorimetry (DSC) analysis (Yoo and Jane 2002). Despite the intensive studies on waxy wheat, few commercial uses for waxy wheat flour have been developed to date.

Conversely, "partial" waxy mutants, lacking one or two GBSSI proteins, appear to be much more practically useful, particularly B-null and AB-null lines (Ishida et al. 2003; Yamamori et al. 1994; Zhao et al. 1998). The amylose contents in these lines are reduced by a small but significant amount, making them particularly suitable for the production of Japanese salted noodles. However, the selection of these partial lines by measuring amylose content requires substantial time and effort by breeders, and we felt that the development of a marker- assisted selection protocol would streamline this process. Our first step towards this goal was the molecular characterization of the mutations in the three waxy genes (Vrinten et al. 1999). This was followed by the development of DNA marker sets that would work well under the same PCR conditions and were co-dominant, making them easy to use in practical breeding programs (Nakamura et al. 2002; Saito et al. 2009). Subsequently, these markers were adapted in Japanese wheat breeding programs, and this essentially marked the beginning of MAS wheat breeding in Japan.

During our work with the waxy mutants, we made several observations which proved useful in our further work on starch modification. First, we noted that early efforts to obtain starch mutants using mutation breeding led to the successful isolation of waxy lines and other starch mutants in diploid plants such maize or rice, but not in wheat. It appeared that wheat had a high resistance to mutagenic treatment, but in fact, mutations likely occurred but were masked by the presence of active homoeologues, and the probability of concurrently mutating all three genes was extremely low. However, combining "partial" null lines represented a simple but effective method of obtaining fully null mutants. Naturally occurring single null mutations can often be identified in material from germplasm databases, and mutagenesis treatments can be used to increase mutation frequency. We also realized that not only fully null mutants but "partial null" lines with mutations in one or two homoeologous genes might prove useful, as was seen for the Waxy genes. The ability to create "partial" mutants can be considered an advantage of working with a hexaploid crop such as wheat. 


\section{High Amylose Wheat (SSIIa Mutant)}

Screening for lines missing the SSIIa proteins was performed by Yamamori et al. (2000) using similar methodology as described above for waxy proteins. After SSII $-A 1,-B 1$ and $-D 1$ null lines were identified, these lines were crossed and a fully null SSIIa mutant was selected. The amylose content in this line was increased by about one-third as compared to wild-type (Yamamori et al. 2000; Shimbata et al. 2005). Although no commercially available HA variety has been released yet, quality studies have shown dramatic changes in flour properties for this type of wheat (Yamamori et al. 2000; Shimbata et al. 2005). HA seed also contains a higher proportion of resistant starch (Yamamori et al. 2006), which is thought to have a beneficial influence on health. Although $H A$ flour used alone does not appear to be suitable for baking or noodle products, combinations of $H A$ and regular flour are giving promising results in ongoing quality tests.

However, our interest has focused more on the "partial null" SSIIa genotypes. We were curious to know if there were significant effects due to the presence of one or two null genes, and if the contributions of the three SSII a genes were different. Again, the use of MAS appeared to be the most efficient way to obtain the desired genotypes. We first characterized the mutations in the three SSIIa genes at the DNA sequence level, then used this information to develop PCR-based markers that were co-dominant and could be multiplexed (Shimbata et al. 2005). After reconstituting the HA line by MAS, we used MAS to develop near-isogenic lines of the eight possible homozygous genotypes for the SSIIa gene, using a leading variety from the southern area of Japan as the recurrent parent.

In less than three years, we obtained $\mathrm{BC}_{5} \mathrm{~F}_{2}$ NIL lines for wild (type 1) and HA (type 8) lines, as well as all "partial" lines (types 2-7). Of the eight lines, only the high amylose line showed significant differences in kernel weight and amylose content as compared to wild-type (Shimbata et al. 2012). However, differences in starch characteristics including chain length distribution, relative viscosity, retrogradation, pasting, and enzymatic hydrolysis properties were observed among partial null lines. Generally, larger effects were observed in lines carrying two null homoeologous genes than in lines carrying a single null gene. Significant differences were also observed among the three lines carrying two null SSIIa genes (Shimbata et al. 2012). These results largely concurred with results from similar studies using doubled haploid lines (Konik-Rose et al. 2007). We were also able to determine that the effect of SSIIa genes on amylopectin synthesis follows the order SSIIa-BI $>$ SSII-DI $\geq$ SSIIa-A1 (Shimbata et al. 2012), which parallels the differential effects of the waxy genes on amylose synthesis $(W x-B 1>W x-D 1 \geq W x-A l)$. 


\section{Sweet Wheat (GBSSI and SSIIa Mutant)}

With two fully null starch mutant lines available, creating a double mutant became possible. Since we had co-dominant markers for wild-type and null alleles of all six genes, selection of the fully null SSIIa/GBSSI double mutant was fairly straightforward. Immature seed of the double mutant showed a higher sugar content, with significant increases in sucrose, glucose, maltotriose and particularly maltose (Nakamura et al. 2006). The seeds of the double mutant appeared to develop normally until seed desiccation began, when the seeds became shrunken, resulting in a large decrease in seed weight, as is seen in some sweet corns. The wheat SSIIa/GBSSI double mutant also resembles sweet corn in terms of its increased sugar content, therefore we refer to it as "sweet wheat" (SW). Mature seed of SW also showed a high sugar content, with increases in fructan and total dietary fiber levels (Shimbata et al. 2011).

The endosperm starch granules of SW are very small and appear degraded, even at earlier stages of seed development, when SW seeds still appear plump (Vrinten et al. 2012). Starch granules from the $H A$ line also have a somewhat deflated appearance in comparison to the normal pancake-like shape found in wild-type granules, suggesting that the lack of functional SSIIa protein may play a major role in this phenotype.

The structure of starch from SW seeds also showed large changes in comparison to starch from wild-type or parental lines. When pasting properties of normal wheat starch are subjected to relative viscosity analysis, a strong peak indicating increased viscosity due to starch swelling is usually observed. However, in SW flour, this peak was essentially absent. As well, the amylopectin from SW seeds had a reduced molecular weight and an increased proportion of short branch chains (Vrinten et al. 2012).

Product tests conducted with SW have indicated that mixing SW flour with regular flour can impart a slightly sweet taste and a crispy texture to baked goods. However, SW flour does not seem to be suitable for use in pasta or noodles. Product tests are still ongoing, and other uses, such as using fresh immature seed as a topping or vegetable, are being considered.

The dramatic changes in starch and seed phenotypes seen in SW can predictably lead to certain problems in the field. For example, SW seed is very light and is therefore easily lost during harvesting, necessitating modification of harvesting machinery. SW also appears to be very susceptible to pre-harvest sprouting, probably due to the high levels of sugar in seeds. 


\section{Expanding Genotype Availability: Combining Mutations}

While the fully null mutants $W x, H A$ and $S W$ have starch characteristics that are of interest to the food and flour milling industries, the dramatic changes resulting from these mutations may lead to some less than desirable characteristics, as outlined above. However, one major advantage in working with wheat is that its hexaploid composition allows us to make more subtle changes in starch characteristics. An example of this is the $W x-B l$ mutation in wheat, which is widely present in lines used in Asian noodles. Although the reduction in amylose content in the $W x-B 1$ mutant is small (approximately $27 \%$ of total starch vs $29 \%$ in wild-type; Yamamori and Quynh 2000), it results in a change in viscoelasticity that imparts improvements in noodle quality.

Using the six available mutations covering the GBSSI and SSIIa genes, 64 homozygous genotypes can be created (Fig. 29.1). By using the co-dominant, "perfect" markers described above, we were able to select all 64 genotypes. We have begun quality testing on these lines, and have identified lines which have significant differences in starch characteristics, yet maintain similar starch levels and seed weights as wild-type lines. Interestingly, we noted that the shrunken phenotype is not limited to the SW genotype (null alleles for all SSIIa and GBSSI genes); certain lines that are null for all SSIIa genes but have a single wild-type GBSSI gene also produce shrunken seeds. When starch granules from all 64 lines were observed using

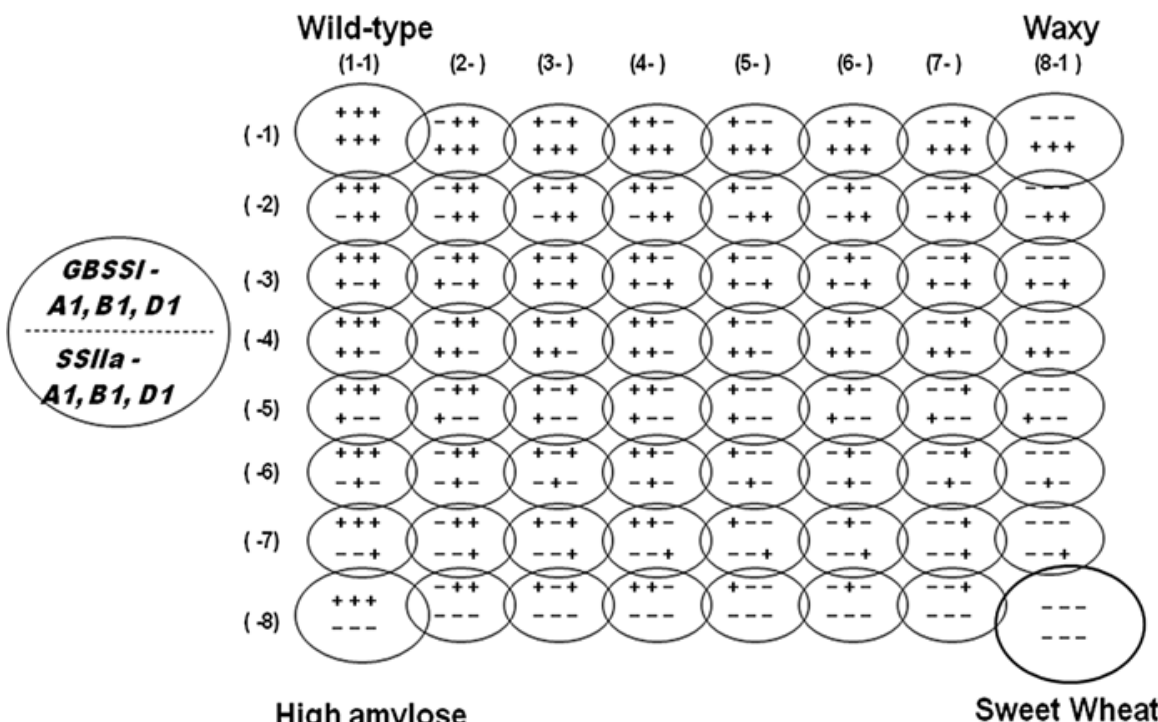

Fig. 29.1 Two- dimensional array of homozygous genotypes developed from GBSSI and SSIIa wild-type and mutant alleles. For each of the six genes, the homozygous wild type allele is indicated by "+" and the homozygous mutant allele by "_-“. Homoeologous genes are indicated as A1, B1 or D1 
scanning electron microscopy, most lines had normal pancake shaped granules, except for lines missing all SSIIa proteins, which had starch granules with flattened shapes. This indicates the importance of amylopectin on starch granule architecture, whereas amylose does not seem to play such a significant role. However, a decrease in the number of functional waxy genes compounded the effects of the null SSIIa genotype.

In contrast to changes in seed shape or starch granule appearance, differences in starch quality characteristics between the lines show a wider range of effects. RVA analysis showed small but significant differences between lines; for example, a line with three null waxy genes and two null SSIIa genes showed a decrease in peak viscosity temperature and an increase in peak viscosity as compared to waxy wheat. Although this is essentially a waxy line, fine-tuning of starch characteristics has resulted from the introduction of two null SSIIa genes, thereby producing a new type of waxy wheat. Similar subtle but significant differences were also observed in other lines, indicating that we have developed a pool of variation for starch quality characteristics simply by making combinations among two sets of homoeologous genes using marker-assisted selection. The chances of developing this amount of variation for starch characteristics naturally in any breeding program would be very small.

Adding a third set of homoeologous genes and making the array "3-dimensional" would increase the number of genotypes exponentially, resulting in a total of 512 genotypes. Although certainly not all of these genotypes would have useful changes in starch characteristics, we expect that some lines would be commercially useful.

\section{Future Directions}

Work with the starch mutations outlined above has demonstrated three main points: (1) Development of fully null mutants of wheat can be achieved by the simple but effective method of identifying individual lines with null mutations in the genes derived from the $\mathrm{A}, \mathrm{B}$ and $\mathrm{D}$ genome, and combining these lines by traditional hybridization. The use of markers greatly facilitates this work. (2) "Partial" mutants, with homozygous null alleles for one or two of a set of three homoeologous genes, can have phenotypes that are significantly different from wild-type. The practical usefulness of these "partial" null lines can exceed that of fully null lines. (3) By creating combinations of homozygous wild-type and null alleles for two or more sets of homoeologous genes, we can create a range of variability for a trait that is unlikely to be present by chance in a breeding program.

The availability of genome sequence information, combined with new methods of mutation detection, significantly reduces the amount of work involved in finding single null mutations in wheat. Although starch synthesis represents a good model for analyzing the effects of null and partial null genotypes in wheat, one can easily see the potential for expanding this methodology to other traits. However, after the detection of single mutants, crossing and analyzing mutant lines remains laborious. Our focus now is on streamlining this process as much as possible. 
Acknowledgments The authors thank Drs. Hisashi Hirano, Makoto Yamamori, and Tsuguo Hoshino for their contributions for early phase of the work. We would like to acknowledge our collaborators, Drs. Pierre Hucl, Robert Graybosch, and Jay-Lin Jane.

Open Access This chapter is distributed under the terms of the Creative Commons Attribution Noncommercial License, which permits any noncommercial use, distribution, and reproduction in any medium, provided the original author(s) and source are credited.

\section{References}

Boyer C, Hannah C (2001) Kernel mutants of corn. In: Hallauer A (ed) Specialty corn. CRC Press, Boca, pp 1-31

Ishida N, Miura H, Noda T, Yamauchi H (2003) Mechanical properties of white salted noodles from near-isogenic wheat lines with different wx protein-deficiency. Starch/Staerke 55:390-396

Konik-Rose C, Thistleton J, Chanvrier H et al (2007) Effects of starch synthase IIa gene dosage on grain, protein and starch in endosperm of wheat. Theor Appl Genet 115:1053-1065

Nakamura T, Yamamori M, Hirano H, Hidaka S (1993) Identification of three Wx proteins in wheat (Triticum aestivum L.). Biochem Genet 31:75-86

Nakamura T, Yamamori M, Hirano H et al (1995) Production of waxy (amylose-free) wheats. Mol Gen Genet 248:253-259

Nakamura T, Vrinten P, Saito M, Konda M (2002) Rapid classification of partial waxy wheats using PCR-based markers. Genome 45:1150-1156

Nakamura T, Shimbata T, Vrinten P et al (2006) Sweet wheat. Genes Genet Syst 81:361-365

Saito M, Vrinten P, Ishikawa G et al (2009) A novel co-dominant marker for selection of the null Wx-B1 allele in wheat breeding programs. Mol Breed 23:209-217

Shimbata T, Nakamura T, Vrinten P et al (2005) Mutations in wheat starch synthase II genes and PCR-based selection of a SGP-1 null line. Theor Appl Genet 111:1072-1079

Shimbata T, Inokuma T, Sunohara A et al (2011) High levels of sugars and fructan in mature seed of sweet wheat lacking GBSSI and SSIIa enzymes. J Agric Food Chem 59:4794-4800

Shimbata T, Ai Y, Fujita M et al (2012) Effects of homoeologous wheat starch synthase IIa genes on starch properties. J Agric Food Chem 60:12004-12010

Vandeputte G, Delcour J (2004) From sucrose to starch granule to starch physical behaviour: a focus on rice starch. Carbohyd Polym 58:245-266

Vrinten P, Nakamura T, Yamamori M (1999) Molecular characterization of waxy mutations in wheat. Mol Gen Genet 261:463-471

Vrinten P, Shimbata T, Yanase M et al (2012) Properties of a novel type of starch found in the double mutant "sweet wheat". Carbohyd Polym 89:1250-1260

Yamamori M, Quynh N (2000) Differential effects of Wx-A1, -B1 and -D1 protein deficiencies on apparent amylose content and starch pasting properties in common wheat. Theor Appl Genet 100:32-38

Yamamori M, Nakamura T, Endo TR (1994) Waxy protein deficiency and chromosomal location of coding genes in common wheat. Theor Appl Genet 89:179-184

Yamamori M, Fujita S, Hayakawa K et al (2000) Genetic elimination of a starch granule protein, SGP-1, of wheat generates an altered starch with apparent high amylose. Theor Appl Genet 101:21-29

Yamamori M, Kato M, Yui M, Kawasaki M (2006) Resistant starch and starch pasting properties of a starch synthase IIa-deficient wheat with apparent high amylose. Crop Pasture Sci 57:531-535 
Yasui T, Sasaki T, Matsuki J, Yamamori M (1997) Waxy endosperm mutants of bread wheat (Triticum aestivum L.) and their starch properties. Breed Sci 47:161-163

Yoo SH, Jane JL (2002) Structural and physical characteristics of waxy and other wheat starches. Carbohyd Polym 49:297-305

Zhao XC, Batey IL, Sharp PJ et al (1998) A single genetic locus associated with starch granule properties and noodle quality in wheat. J Cereal Sci 27:7-13 